RESPIRATORY RESEARCH

\title{
Research outputs in respiratory medicine
}

\author{
I Rippon, G Lewison, M R Partridge
}

Thorax 2005;60:63-67. doi: 10.1136/thx.2004.031229

See end of article for authors' affiliations .....................

Correspondence to: Professor M R Partridge, Department of Respiratory Medicine, Imperial College London, Charing Cross Campus, London W6 8RP, UK; m.partridge@ imperial.ac.uk

Received 6 July 2004 Accepted 4 August 2004
Background: There is currently little information regarding how much the distribution of research activity in respiratory medicine reflects the interests of its clinicians and scientists, the disease burden in any country, or the availability of funding.

Methods: A total of 81419 respiratory medicine publications identified in the Science Citation Index for the years 1996-2001 were assigned to 14 subject areas (mainly based on title words) and to 15 OECD countries. Outputs were compared with a nation's disease burdens and, for the UK, the sources of research funding were investigated.

Results and conclusions: Overall, Finland, Canada, Spain and the UK had the greatest relative commitment to respiratory medicine research expressed as a ratio of their share of world biomedical research. The largest subject areas were asthma, lung cancer, and paediatric lung disease, each with over 1400 papers published per year. Australia and Canada led in relative commitment to sleep research and Sweden and Finland led in research on asthma. Australia and the UK produced significant numbers of publications on cystic fibrosis (CF) but Finland produced few. The Netherlands has a strong output on chronic obstructive pulmonary disease (COPD), France and the UK on diffuse parenchymal lung disease (DPLD), and Finland dominated occupational lung disease research but had few publications on HIV/AIDS where Spain proportionately produced most. Finland and Australia had strong outputs in paediatric lung disease research. For most subject areas the research output of a country correlated poorly with disease burden. In the UK, lung cancer research appeared unduly low in relation to the number of deaths and COPD outputs were low compared with those for asthma. However, correlations were positive for the burden of CF and pulmonary complications of HIV/AIDS which explains, for example, the low outputs in these subject areas from Finland. The strong performance in CF research in the UK is likely to reflect significant charitable funding, while sleep research, pulmonary circulatory disease, and DPLD had little stated external funding or sponsorship.
T he priority attached to clinical services for those with respiratory illness varies from country to country. Some such as Australia and Finland have national initiatives for asthma, ${ }^{1}$ while others such as the UK have priority arrangements for cancer, including lung cancer. The World Health Organization (WHO) has proposed strategies for the prevention and control of chronic respiratory disorders. ${ }^{23}$ However, it is unclear whether research spending follows clinical priorities and whether such spending parallels the burden of disease in individual countries. We have sought to analyse the outputs of research papers in respiratory medicine from around the world, to compare them with the burden of disease in individual countries and, for UK papers, to determine the sources of funding for the research.

It is debatable whether research activity should reflect the relative disease burden, either globally or within a country. Some basic research may also benefit several disease areas. Nevertheless, there has been increasing discussion as to whether research budgets should take account of the relative burden of disease and the numbers of patients affected. In the USA increased financial resources given to the National Institutes of Health have led patient advocacy groups to demand a more "equitable" share of research grants. ${ }^{4}$ Globally, there are gaps between the resources devoted to diseases that affect the rich and the poor. ${ }^{5-9}$ Recent debate has concerned whether greater funding of cell and molecular biology has delivered what was promised to respiratory medicine. ${ }^{10}$ Such debate can only be meaningfully serviced by better understanding of what determines research priorities and by having available data on the distribution and funding of current research outputs.

\section{METHODS}

Articles and reviews related to respiratory medicine for the years 1996-2001 were identified and downloaded from the Science Citation Index (SCI) (Institute for Science Information (ISI)) by means of a specially designed "filter" based on the names of specialist journals and title keywords. ${ }^{11}$ The filter was estimated to have a specificity (or precision) of 0.97 and a sensitivity (or recall) of 0.89 , so that it gave a much better coverage of the sub-field than reliance on journal sets. Papers from 15 selected OECD countries were then identified on the basis of their addresses, all of which are recorded in the SCI. These countries, together with their digraph ISO codes used in the tables and figures, are shown in table 1 .

Each downloaded paper was then allocated to one of 14 subject areas listed in table 2, with trigraph (three letter) codes used also in table 3 by means of a similar process. As an example of the process, title words or parts of words used by themselves to identify papers in "apnoea and other sleep related breathing disorders" included: apnea, apneic, apnoe*, apnoeic, daytime sleepiness, Epworth, hypopnea, hypopnoea, polysomnographic, snorer, snoring and somnolen*. (The suffix asterisk implied that anything following the asterisk would be included if the preceding letters were present.) Title words or parts of words occurring in combination were also used to identify papers. Examples from the same subject area were: breathing+sleep, hypoventilation+obesity, obstruct*+ sleep, sleep+ventilat*, sleep+upper airway, and CPAP + (compliance or sleep or therapy or treatment).

A similar scheme was used to allocate papers to the other subject areas; for some, specialist journals were also used. 
Table 1 List of 15 OECD countries used in the analysis with their annual outputs of respiratory medicine research papers in 1996-2001 (Resp), their relative presence in the world literature (\% of world), their relative presence in world biomedical papers (Biomed), and the ratio between these percentages (relative commitment)

\begin{tabular}{|c|c|c|c|c|c|}
\hline \multirow[b]{2}{*}{ Country } & \multirow[b]{2}{*}{ Code } & \multicolumn{2}{|l|}{ Resp } & \multirow{2}{*}{$\frac{\text { Biomed }}{\% \text { of world }}$} & \multirow[b]{2}{*}{ Relative commitment } \\
\hline & & Papers/year & $\%$ of world & & \\
\hline Australia & $\mathrm{AU}$ & 385 & 2.8 & 2.7 & 1.04 \\
\hline Austria & AT & 147 & 1.1 & 1.1 & 0.95 \\
\hline Belgium & $\mathrm{BE}$ & 224 & 1.7 & 1.6 & 1.03 \\
\hline Canada & CA & 754 & 5.6 & 4.7 & 1.17 \\
\hline Denmark & DK & 173 & 1.3 & 1.4 & 0.90 \\
\hline Finland & $\mathrm{FI}$ & 206 & 1.5 & 1.2 & 1.21 \\
\hline France & FR & 827 & 6.1 & 6.3 & 0.96 \\
\hline Germany & $\mathrm{DE}$ & 907 & 6.7 & 8.3 & 0.80 \\
\hline Italy & IT & 600 & 4.4 & 4.7 & 0.94 \\
\hline Netherlands & NL & 462 & 3.4 & 3.3 & 1.04 \\
\hline Spain & ES & 411 & 3.0 & 2.8 & 1.09 \\
\hline Sweden & SE & 390 & 2.9 & 2.8 & 1.04 \\
\hline Switzerland & $\mathrm{CH}$ & 276 & 2.0 & 2.2 & 0.94 \\
\hline UK & UK & 1486 & 10.9 & 10.2 & 1.08 \\
\hline USA & US & 5312 & 39.1 & 39.5 & 0.99 \\
\hline World & & 13570 & 100.0 & 100.0 & 1.00 \\
\hline
\end{tabular}

Any paper that could not be allocated to one of the 13 defined subject areas was classified as miscellaneous; this classification clearly contained many papers reporting research in basic mechanisms.

Each country's relative commitment to research in respiratory medicine overall and to the individual subject areas was calculated as the ratio between its percentage presence in the subject and its percentage presence in biomedical research overall. ${ }^{12-14}$ Thus, Canada publishes $4.7 \%$ of biomedical research overall but $5.6 \%$ of research in respiratory medicine; its relative commitment to the subject is therefore $5.6 / 4.7=1.19$ (the percentage figures have been rounded, so the above calculation is not exact). These relative commitments were then compared with the known death rates per 100000 population (averages for males and females) for those disease areas where it was possible to obtain data from WHO national mortality tables ${ }^{15}$ by means of scattergraphs. For asthma the comparison was with childhood prevalence taken from the ISAAC study ${ }^{16}$ and, for cystic fibrosis, with the prevalence of the disease at birth. ${ }^{17}{ }^{18}$

The UK papers were matched with papers in the Research Outputs Database ${ }^{12}$ which lists all UK biomedical papers whose funding acknowledgements have been inspected in libraries to determine their sources of support. These were grouped into main sectors as follows:

- UK government, including departments and agencies such as the Medical Research Council, Department of Health, and Biotechnology and Biological Sciences Research Council.

- UK private-non-profit, including charities such as the British Lung Foundation, National Asthma Campaign, Cystic Fibrosis Trust, Cancer Research UK, and foundations such as the Wellcome Trust.

- Industry - mainly pharmaceutical companies.

- International, including the European Commission, the WHO and the US National Institutes of Health.

\section{RESULTS}

\section{World papers}

A total of 81419 respiratory medicine research papers were identified between 1996 and 2001. Table l shows the mean annual outputs for the 15 OECD countries used for the analysis with, for comparison, their annual percentage presences in respiratory medicine and in biomedicine, and

Table 2 Respiratory medicine research subject areas and their outputs: 1996-1998, 1999-2001, the ratio between them, the total for 1996-2001, and the percentage of all respiratory medicine research outputs

\begin{tabular}{llrrrrr}
\hline Subject area & Code & $\mathbf{9 6 - 9 8}$ & $\mathbf{9 9 - 0 1}$ & Ratio & Total & $\%$ \\
\hline Miscellaneous respiratory research & MIS & 16818 & 16899 & 1.00 & 33717 & 41.4 \\
Asthma and other allergic lung diseases & AST & 5325 & 5973 & 1.12 & 11298 & 13.9 \\
Cancer & CAN & 3942 & 4386 & 1.11 & 8328 & 10.2 \\
Children's lung diseases & PED & 3952 & 4109 & 1.04 & 8061 & 9.9 \\
Pneumonia & PNE & 2238 & 2907 & 1.30 & 5145 & 6.3 \\
Tuberculosis & TUB & 2226 & 2608 & 1.17 & 4834 & 5.9 \\
Viral respiratory infections incl influenza & INZ & 1772 & 1786 & 1.01 & 3558 & 4.4 \\
Pulmonary circulatory disease & PCD & 1185 & 1221 & 1.03 & 2406 & 3.0 \\
Cystic fibrosis & CFB & 1222 & 1176 & 0.96 & 2398 & 2.9 \\
Occupational lung diseases & OCC & 1145 & 1095 & 0.96 & 2240 & 2.8 \\
Chronic obstructive pulmonary disease & COP & 859 & 1109 & 1.29 & 1968 & 2.4 \\
Apnoea and sleep related breathing disorders & APN & 939 & 1024 & 1.09 & 1963 & 2.4 \\
Diffuse interstitial lung disease & DIL & 803 & 801 & 1.00 & 1604 & 2.0 \\
Pulmonary manifestations of HIV/AIDS & HIV & 641 & 529 & 0.83 & 1170 & 1.4 \\
Respiratory disease total & RESPI & 39663 & 41756 & 1.05 & 81419 & 100 \\
\hline
\end{tabular}


Table 3 Relative commitments of 15 OECD countries to different subject areas (other than miscellaneous) within respiratory medicine research in relation to their relative presence in biomedical research

\begin{tabular}{|c|c|c|c|c|c|c|c|c|c|c|c|c|c|c|c|c|}
\hline Subject & & AT & AU & BE & CA & $\mathrm{CH}$ & $\mathrm{DE}$ & DK & ES & FI & FR & IT & NL & SE & UK & US \\
\hline Respiratory disease total & RESPI & 0.95 & 1.04 & 1.03 & 1.17 & 0.94 & 0.80 & 0.90 & 1.09 & 1.21 & 0.96 & 0.94 & 1.04 & 1.04 & 1.08 & 0.99 \\
\hline Apnoea and sleep related breathing disorders & APN & 0.71 & 2.16 & 1.46 & 1.92 & 0.71 & 1.04 & 0.25 & 1.17 & 1.71 & 1.15 & 0.63 & 0.29 & 1.63 & 0.88 & 0.95 \\
\hline Asthma and other allergic lung diseases & AST & 1.40 & 1.81 & 1.15 & 1.43 & 0.89 & 0.62 & 1.32 & 0.99 & 1.92 & 0.73 & 1.09 & 1.66 & 2.08 & 1.71 & 0.76 \\
\hline Cancer & CAN & 0.84 & 0.50 & 1.24 & 0.74 & 0.77 & 0.87 & 0.82 & 0.92 & 1.51 & 0.98 & 1.64 & 1.10 & 0.72 & 0.68 & 0.88 \\
\hline Cystic fibrosis & CFB & 0.91 & 2.01 & 1.41 & 1.62 & 1.26 & 0.89 & 1.36 & 0.54 & 0.13 & 1.59 & 0.85 & 0.98 & 0.74 & 1.98 & 0.99 \\
\hline Chronic obstructive pulmonary disease & $\mathrm{COP}$ & 0.71 & 0.45 & 1.81 & 1.67 & 1.08 & 0.52 & 1.33 & 1.88 & 0.98 & 0.78 & 1.96 & 2.61 & 0.74 & 1.46 & 0.75 \\
\hline Diffuse interstitial lung disease & DIL & 0.82 & 0.55 & 0.51 & 0.46 & 0.58 & 0.94 & 0.49 & 0.89 & 1.10 & 1.34 & 0.93 & 0.84 & 0.91 & 1.23 & 0.74 \\
\hline Pulmonary manifestations of HIV/AIDS & HIV & 0.60 & 0.53 & 0.43 & 0.58 & 1.62 & 0.25 & 1.45 & 2.64 & 0.00 & 1.24 & 1.70 & 0.86 & 0.53 & 1.07 & 1.15 \\
\hline Viral respiratory infections including influenza & INZ & 0.51 & 1.46 & 0.60 & 0.52 & 0.83 & 0.62 & 0.83 & 1.05 & 1.26 & 0.57 & 0.35 & 1.31 & 0.90 & 1.32 & 1.27 \\
\hline Occupational lung diseases & OCC & 0.58 & 1.05 & 1.51 & 1.57 & 0.62 & 0.85 & 0.82 & 1.33 & 4.11 & 0.86 & 1.56 & 1.36 & 1.67 & 1.15 & 0.89 \\
\hline Pulmonary circulatory diseases & PCD & 1.70 & 0.66 & 0.86 & 1.09 & 1.27 & 0.99 & 0.38 & 0.60 & 0.60 & 1.31 & 0.64 & 0.89 & 0.69 & 0.76 & 1.03 \\
\hline Pneumonia & PNE & 0.63 & 0.84 & 0.56 & 1.06 & 1.00 & 0.99 & 1.22 & 2.27 & 2.23 & 1.31 & 0.29 & 1.12 & 0.92 & 0.74 & 1.01 \\
\hline Tuberculosis & TUB & 0.29 & 0.66 & 1.03 & 0.53 & 1.40 & 0.47 & 1.40 & 1.74 & 0.35 & 0.90 & 0.74 & 0.88 & 0.51 & 0.80 & 0.89 \\
\hline
\end{tabular}

Values $<0.25$ are shaded dark; values $0.25-0.5$ are lightly shaded; values $>2$ are shown in bold; values $>4$ are in bold and boxed.

the ratio between them. Overall, Finland, Canada, Spain, and the UK are seen to be relatively strong in respiratory medicine (fig 1).

The breakdown of publications in each of the 14 subject areas is shown in table 2 for two 3 year periods. (The subject area "bronchitis (except chronic)" proved to be so small that

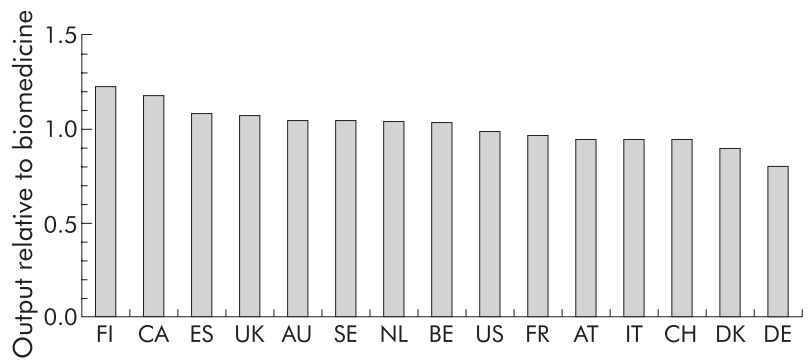

Figure 1 Relative commitment to research in respiratory medicine of 15 OECD countries, 1996-2001 (relative presence in respiratory medicine divided by relative presence in all biomedicine).

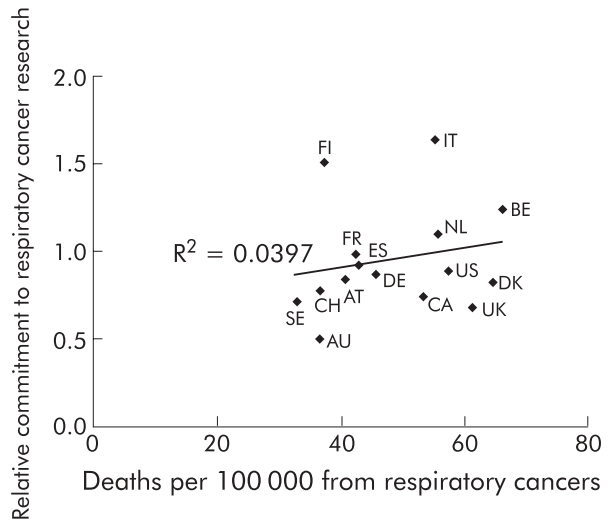

Figure 2 Comparison of relative commitment to respiratory cancer research, 1996-2001, with death rates from respiratory cancers (1995) for 15 OECD countries (country codes as shown in table 1). no further analysis was applied to this group of papers.) After "miscellaneous", asthma, lung cancer and paediatric lung diseases accounted for the three largest numbers of papers.

For each of the respiratory subject areas we calculated a country's relative commitment to that subject area as a ratio of its presence in biomedicine. The results are shown in table

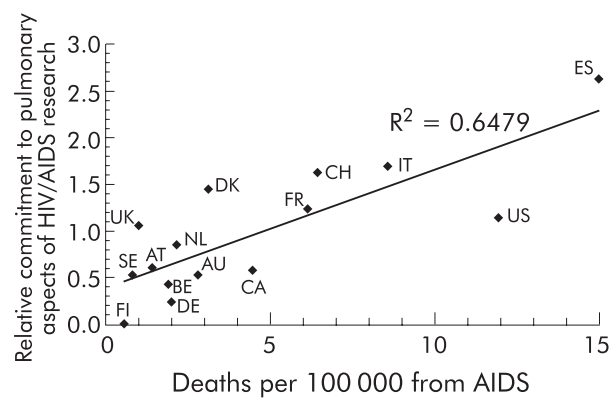

Figure 3 Comparison of the relative commitment to pulmonary manifestations of HIV/AIDS research (1996-2001) with death rates from HIV/AIDS (1995) for 15 OECD countries (country codes as shown in table 1).

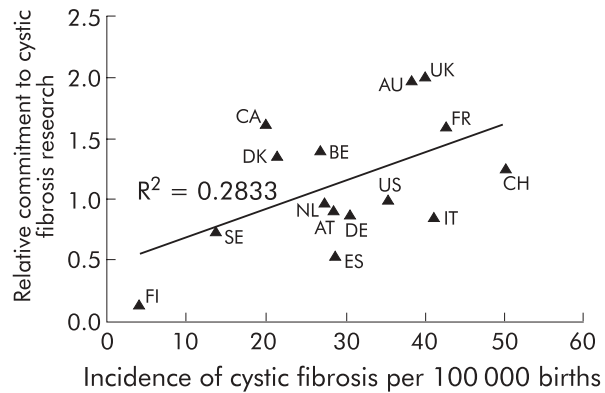

Figure 4 Comparison of the relative commitment to cystic fibrosis research (1996-2001) with incidence of the disease (1995) for 15 OECD countries (country codes as shown in table 1). 
3. Australia, Canada, Finland and Sweden are seen to have a strong publication presence in sleep related breathing disorders. Sweden, Finland, Australia and the UK are well represented in research publications related to asthma. Australia and the UK appear to have a strong commitment to cystic fibrosis research, and the Netherlands a very strong commitment to chronic obstructive pulmonary disease. France and the UK are relatively well represented in the small number of research publications output related to diffuse interstitial lung disease, while Spain is particularly strong on research in the pulmonary manifestations of HIV/ AIDS. Australia, the UK, the Netherlands, the USA and Finland are major producers of research publications in viral infections, including influenza (but excluding HIV). Finland dominates research outputs in occupational lung diseases and in paediatric pulmonary research (with Australia) and pneumonia (with Spain). Spain also has a significant research publication output in the field of tuberculosis.

We found relating research outputs to the burden of the disease for each country to be difficult, especially for disease areas such as sleep related breathing disorders where there are very poor records of the burden of disease. In most subject areas correlations were weak. As an example, fig 2 shows the relative commitment to respiratory cancer research related to death rate. Italy and Finland can be seen to do more than the mean for the other countries. The UK output is quite low, perhaps especially in view of its high lung cancer mortality rates. Correlations between research commitment and death rates were effectively zero for tuberculosis and for asthma and COPD, although there was a weak positive correlation with asthma prevalence (data not shown). For publications on pulmonary complications of HIV and AIDS there was a positive correlation with mortality (fig 3), with Spain having

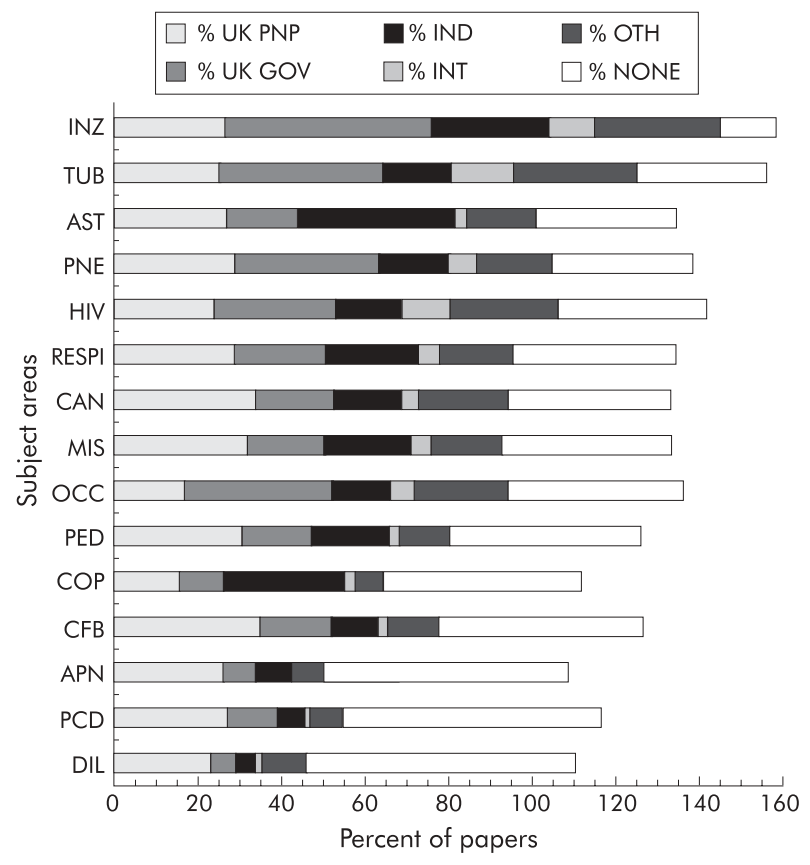

Figure 5 Percentages of respiratory medicine papers funded by the main funding sectors: UK Charities and Foundations (PNP, e.g. Cancer Research UK), UK Government sources (GOV, e.g. Medical Research Council, Department of Health), Pharmaceutical Industry (IND), International funders (INT) and other (OTH). The blank rectangles represent papers without any stated funding source. The codes for the subject areas are given in table 2. The code RESPI relates to the sum of all respiratory publications and the miscellaneous category (MIS) reflects basic science research that could not be attributed to any one disease area. (Note: funding totals exceed $100 \%$ as many papers acknowledge funding from several sectors in parallel.). the highest death rate (among the countries studied) and the highest research commitment. The USA has a high death rate but only a modest research publication output in this subject area. For cystic fibrosis the UK and Australia had the highest relative commitment but also a high incidence of the disease. Finland has the lowest output and the lowest incidence of the disease (fig 4).

Funding and geographical distribution of UK research Declared sources of sponsorship and funding for UK research papers published in the years 1996-2000 were analysed. Of 7401 papers, $7048(95 \%)$ had been inspected and 4307 of them $(61 \%)$ had one or more funding acknowledgements. This is lower than for total biomedical research overall in the UK. ${ }^{12}$ The leading sources of declared research sponsorship are shown in table 4.

Figure 5 shows that the amount of stated research sponsorship varies by disease area, with influenza followed by tuberculosis, asthma, pneumonia and HIV/AIDS having the most declared support and pulmonary circulatory disease and diffuse parenchymal lung disease having the least. There were also variations in the amount of support from different sources. The highest levels of government support are in influenza $(50 \%)$, tuberculosis $(39 \%)$, and occupational lung diseases (36\%); charities and foundations favour cystic fibrosis (35\%) and respiratory cancer (34\%); and the pharmaceutical industry supports primarily asthma (37\%), COPD, and influenza (28\%). The percentages of support from the individual sectors, together with those papers without financial acknowledgements, add up to more than 100\% because many papers have funding from several sectors in parallel.

\section{DISCUSSION}

This report describes an observational tool, use of which has provided an interesting insight into the relative volumes of published research output on a variety of respiratory conditions in different countries. Most countries appear to commit to research into respiratory disease at approximately the same relative level as in biomedical research overall, although Finland and Canada, for example, seem to do rather more respiratory research and Germany rather less. Different countries will be interested in different aspects but, in the

Table 4 Leading sources of support for UK respiratory medicine research, 1996-2000

\begin{tabular}{lrr}
\hline & No of \\
Funding source & papers & \multicolumn{1}{c}{$\%$} \\
\hline UK government, of which: & 1558 & 22.1 \\
Medical Research Council (MRC) & 792 & 11.2 \\
Department of Health (DoH) & 270 & 3.8 \\
Biotechnology \& Biological Sciences Research & 136 & 1.9 \\
Council & & \\
UK private-non-profit organisations, of which: & 2047 & 29.1 \\
Wellcome Trust & 639 & 9.1 \\
National Asthma Campaign & 297 & 4.2 \\
British Lung Foundation & 200 & 2.8 \\
British Heart Foundation & 165 & 2.3 \\
Cystic Fibrosis Research Trust & 126 & 1.8 \\
Cancer Research Campaign (now part of Cancer & 103 & 1.5 \\
Research UK) & 1532 & 21.7 \\
Industry, of which: & 451 & 6.4 \\
GlaxoWellcome plc & 161 & 2.3 \\
AstraZeneca plc & 1405 & 19.9 \\
International and foreign organisations, of which: & 231 & 3.3 \\
European Union & 225 & 3.2 \\
US National Institutes of Health & 2739 & 38.9 \\
No funding acknowledged & 7046 & 100.0 \\
Total papers & & \\
\hline & & \\
&
\end{tabular}


UK, it can be seen that lung cancer is relatively underresearched considering the very high burden of the disease in this country. On the other hand, cystic fibrosis research is particularly strong relative to the clinical burden of the disease, and a sizeable part of the funding comes from the Cystic Fibrosis Trust, a charity which is the acknowledged source of sponsorship on 99 out of 379 UK papers (26\%). Another apparent research strength in the UK is asthma where there is a relative commitment 1.7 times that in biomedicine overall. Again the charities and foundations play a substantial part, as does the pharmaceutical industry, with one large company (GlaxoWellcome* plc) acknowledged on $13 \%$ of the UK output (205 of 1595 papers inspected). This company also funded $14 \%$ of UK influenza research and $11 \%$ (23 out of 216 inspected papers) in COPD. Of the 431 inspected papers concerning UK respiratory cancer research, the charity Cancer Research UK† supported over $22 \%$ of the UK output. Government support for cancer research was rather low at 19\%, being proportionately less than its support for influenza, tuberculosis, pneumonia, HIV and occupational lung diseases.

Some disease areas are undersupported in terms of both government research spending and sponsorship by charities, foundations, and the pharmaceutical industry. For example, it may be seen that, whereas research into infectious diseases is rarely published without acknowledgement of a funding source, the majority of research publications related to apnoea and sleep related breathing disorders, pulmonary circulatory disorders, and diffuse parenchymal lung disease have no outside stated sponsorship. There may be a number of explanations for this. These may be areas that are therapeutically unattractive to major pharmaceutical company sponsors or they may be areas in which there are no disease-specific charities. They may represent areas in which there is an absence of data regarding the burden of disease and the need for research may therefore not have been appreciated by funding bodies. Nevertheless, these are important areas-see, for example, recent work on sleep apnoea. ${ }^{19} 20$

Research publication outputs may not necessarily accurately reflect all work being done in that area. For example, work done by the pharmaceutical industry may not be published for reasons of commercial confidentiality. Outputs may similarly not necessarily equate to quality, but a cursory glance at Citation Indices does suggest an association between output and the more frequently cited journals, but further work may be needed to elucidate this.

The availability of data is an important starting point for future strategic planning. This observational study of international research outputs in different disease areas has shown that there are variations in the importance attached to respiratory research in different countries, and that that importance does not always relate to the burden of disease in those countries. In some countries the burden of disease-for example, lung cancer in the UK-would suggest that a greater effort is needed into research in that area. It is also important for the sponsors of research (both governmental and private-non-profit) to be aware that "new" areas such as sleep related breathing disorders and those without the likelihood of attracting pharmaceutical company funding should not be overlooked.

\section{ACKNOWLEDGEMENTS}

The authors thank Professor Sally Davies, National Health Service Research \& Development Lead for London and for Respiratory Medicine for permission to publish this paper.

\section{Authors' affiliations}

I Rippon, G Lewison, Department of Information Science, City University, London ECIV OHB, UK

M R Partridge, Faculty of Medicine, NHLI Division at Charing Cross Hospital, Imperial College London, London W6 8RP, UK

This work was commissioned and funded by the UK Department of Health.

\section{REFERENCES}

1 Partridge MR. The profile of respiratory conditions: why government action is needed. Thorax 2001;56:744-5.

2 World Health Organization. WHO consultation on the development of a comprehensive approach for the prevention and control of chronic respiratory disorders. Publication No WHO/NMH/MNC/CRA/01.1. Geneva: WHO, 2001

3 World Health Organization. Implementation of the WHO strategy for prevention and control of chronic respiratory diseases. Publication No WHO/ MNC/CRA/02.2. Geneva: WHO, 2002.

4 Anon. US rally calls for doubling of funds to cancer institute. Nature 1998;395:424

5 Ahmad K. Report reveals serious imbalance in global research funding. Lancet 2000;355:1706.

6 O'Neale J. Research does not reflect global disease burden. BMJ 2000;320: 1228.

7 Abbasi K. Progress is slow in narrowing the health research divide. BMJ 2001;323:886.

8 Ramsay S. No closure in sight for the 10/90 health-research gap. Lancet 2001;358:1348.

9 Ferriman A. Where there is despair, hope. BMJ 2002;325:1194.

10 Lenfant CL, Macklem PT. Pro/con editorial. Greater funding of cell and molecular biology has (has not) delivered what was promised to respiratory medicine. Am J Respir Crit Care Med 2004;169:437-40.

11 Lewison G. The definition and calibration of biomedical subfields. Scientometrics 1999;46:529-37.

12 Webster B, Lewison G, Rowlands I. Mapping the landscape II: Biomedical research in the UK, 1989-2002. London: City University, 2004.

13 Dawson G, Lucocq B, Cottrell R, et al. Mapping the landscape: national biomedical research outputs 1988-95. Policy Report No 9. London: The Wellcome Trust, 1998

14 Lewison G, Paraje G. The classification of biomedical journals by research level. Scientometrics 2004;60:145-57.

15 World Health Organization. WHO mortality database. Available at: http:// www3. who.int/whosis/menu.cfm?path = mort (last updated 25 February 2003).

16 Asher Ml, et al. Worldwide variations in the prevalence of asthma symptoms: the International Study of Asthma and Allergies in Childhood (ISAAC). Eur Respir J 1998;12:315-35.

17 Bobadilla JL, Macek M, Fine JP, et al. Cystic fibrosis: a worldwide analysis of CFTR mutations - correlation with incidence data and application to screening. Human Mutat 2002;19:575-606.

18 Lucotte G, Hazout S, de Braekeleer M. Complete map of cystic fibrosis mutation DF508 frequencies in western Europe and correlation between mutation frequencies and incidence of disease. Human Biol 1995;67:797-803.

19 Teran Santos J, Jimenez Gomez A, Cordero Guevara J. The association between sleep apnea and the risk of traffic accidents. N Engl J Med 1999;340:847-51

20 Young T, Peppard PE, Gottlieb DJ. Epidemiology of obstructive sleep apnea: a population health perspective. Am J Respir Crit Care Med 2002;165:1217-39.
*For the period of the analysis GlaxoWellcome plc had not yet merged with SmithKlineBeecham plc.

tThis was formed in 2002 by a merger of the Cancer Research Campaign and the Imperial Cancer Research Fund. 